\title{
From Biological Rhythms to the Default Mode Network: What Lies beneath the Tip of the Iceberg of Mind?
}

\author{
Ravinder Jerath*, Connor Beveridge \\ Charitable Medical Healthcare Foundation, Augusta, GA, USA \\ Email: *Rj605r@aol.com
}

How to cite this paper: Jerath, R. and Beveridge, C. (2019) From Biological Rhythms to the Default Mode Network: What Lies beneath the Tip of the Iceberg of Mind? World Journal of Neuroscience, 9, 262-281. https://doi.org/10.4236/wjns.2019.94020

Received: September 13, 2019

Accepted: October 18, 2019

Published: October 21, 2019

Copyright (อ 2019 by author(s) and Scientific Research Publishing Inc. This work is licensed under the Creative Commons Attribution International License (CC BY 4.0).

http://creativecommons.org/licenses/by/4.0/

\section{(c) (i) Open Access}

\begin{abstract}
Our conscious day-to-day self is often described as the "tip of the iceberg" of a much greater cognitive system. The edge of the water divides the phenomenal self from the sub/unconscious underlying it. Similar to an iceberg, the unconscious activity below the water vastly outweighs the conscious activity above it. What exactly lies beneath the surface of this murky water is a tantalizing topic of research and theory. The current research predominantly focuses on the physiology of the brain and the default mode network has been identified as an intrinsic mode of functioning. It is well known that autonomic nervous system sympathovagal balance orchestrated by the central autonomic network is strongly associated with modulation of cardiac, respiratory rate and other visceral physiological activity. In this article, we use existing research and a novel theory to tie together the default mode network, the autonomic nervous system, and non-neural physiology to describe a hypothesis on a greater biological system from which intrinsic brain activity may be founded. This hypothesis is that intrinsic brain activity and connectivities are significantly founded on activity of the body. We review how cardiorespiratory and other rhythms and electrical activity of the body may modulate and even underlie fundamental activity of the human brain and ultimately the mind. A more holistic biological system that could interface the brain and body via mechanisms such as neurovascular coupling would more accurately describe the nature of neural systems. Greater knowledge on the association and interface of brain and body via isomorphic physiologic counterparts to mind may carry profound implications in understanding intrinsic activity of the brain, consciousness, mind, and mental illness.
\end{abstract}

\section{Keywords}

Default Mode Network, Embodied Cognition, Mind, Consciousness 


\section{Introduction}

Embodied cognition is a set of hypotheses that the brain is not the sole cognitive resource and that our bodies play a significant role in various aspects of mind [1]. Theories of embodied cognition describe how at least some cognitive processes are best conceptualized as a dynamic interaction between neural activity of the brain and somatic activity of the body [2]. In addition to the extension of cognitive facilities to the body, this approach has been considered radical because it rejects the "mind as a computer" metaphor [3]. In describing the intimate relationship between neural activities of the default mode network (DMN), the autonomic nervous system (ANS), non-neural physiology of the body, we extend this approach to intrinsic cognitive systems, propounding and supporting the hypothesis that processes of the body significantly underlie the emergence of mind. In doing so, the massive body of ice (un/subconscious physiology) under the tip of the iceberg (conscious self) of mind may be revealed.

The central nervous system is known for its capacity to learn, perceive, and make decisions; however, neural activity is not the only biological processes which implement informational systems from which cognitive abilities emerge [4]. For example, non-neural animal cells, tissues, and organs have been shown to sometimes behave similar to neural networks [5], demonstrating the use of memory [6] [7] [8], computation [9], and decision making often via electrical mechanisms [4] [10]. Neurons in the body also may do high level processing; neurons in the skin perform edge detection [11]. The electrical mechanisms used by neurons evolved from cellular properties which themselves evolved long before neurons did [12] [13]. Thus in the evolutionary sense, non-neural activity underlies neural activity. In this article, we extend the assertion made by many that visceral activity of the lungs [14] [15] [16] [17] [18], heart, gut, and their peripheral, autonomic nervous system counterparts [19] may underlie or at least significantly influence basic bioelectric activity in the brain [20] of which a greater, global bioelectric architecture has been proposed to be isomorphic to phenomenal consciousness [21] [22] [23]. Phenomenal consciousness is best defined as "pure experience" [24]. In supporting this assertion that non-neural activity may underlie neural activity we review here the literature which has analyzed this possibility.

Patterns in breathing have been believed by many since ancient times to be a powerful force in modulating and medically treating the mind as well as fostering a variety of spiritual states with many religions and ancient philosophies identifying breath with the soul [25]. Modern medical research is beginning to reveal scientifically the power breathing can have on the mind and body [26] Breathing techniques are even used by the military to maintain composure and reduce stress [27]. We have previously described a layered hierarchy of mind of which cardio-respiratory and DMN network activity is at the foundation [28] (Figure 1). Researchers with predominant theories on the "tip of the iceberg" of mind have described the relatively massive unconscious system underlying it to 


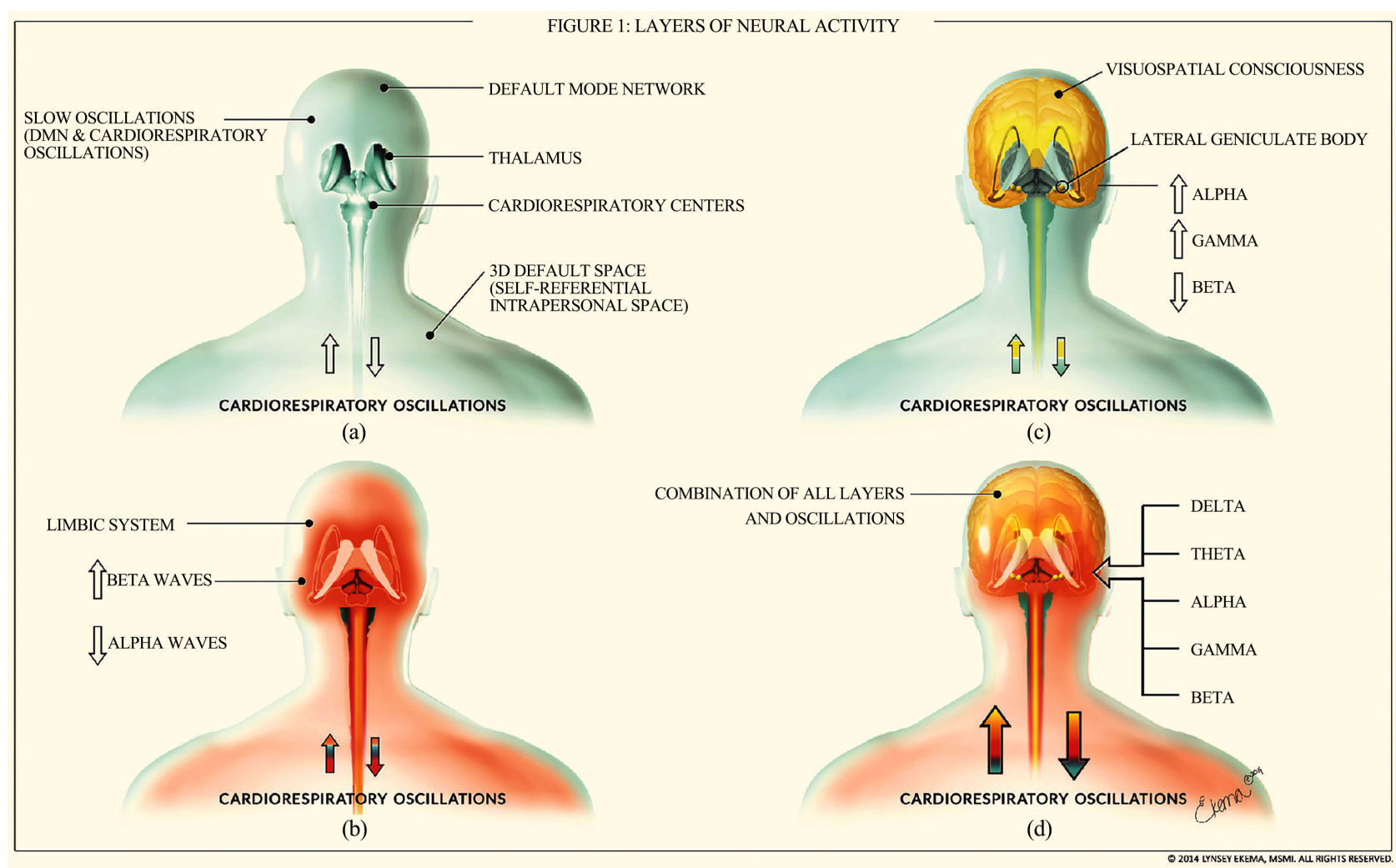

Figure 1. A Model of Layered Activity. This image illustrates a theory on a hierarchical nature of bioelectric activity of mind which highlights the idea that bodily activity lies at the base of this hierarchy. Panel (a) is of particular interest for this article as it illustrates the base layer of slow neural oscillations of the default mode network and cardiorespiratory activity underlying it. This creates a foundation for all other layers of oscillatory activity and is depicted by the blue coloring. Slower oscillations do indeed underlie and entrain faster ones in the brain and so it may follow that even oscillations of the body may do the same. The 3D Default Space is labeled in this panel which is a theoretical phenomenal space isomorphic to a bioelectric framework which not only spans the brain but extends to the body. Panels (b) and (c) show the higher layers of the hierarchy which consist of increasing frequencies of oscillations and more complex cognitive systems. Panel (d) combines these layers to form the sum of human neural activity consisting of all neural and physiological oscillations. Previously published in [28], permission by CC-BY.

be composed of the basic physical operations of neurons [21] while the glia, vessels, etc. are most often left out [29]. In this article, we propound this unconscious system includes basic cardio, respiratory, and other rhythms of the body in a holistic manner. Thus, we aim to reassess the nature of bioelectric neural oscillations and intrinsic networks by linking them to physiological rhythms from the body.

The understanding of how fundamental characteristics of neural activity arise from fundamental body rhythms could lead to new treatments for and categorizations of mental and neurophysiological disorders as well as the development of new technologies. The current organization of mental disorders as laid out in the Diagnostic and Statistical Manual of Mental Disorders, or DSM, is incomplete as it is far removed from the underlying psycho-neuropathological processes [30] [31] [32], instead deriving diagnosis from subjective reports and psychiatrist observations of behavior [33] [34]. It is thus even farther removed without considering what else (body rhythms) lies beneath the tip of the iceberg of common 
psychopathology. A more recent framework, Research Domain Criteria, or RDoc, identifies "domains" or brain systems implicit in different psychiatric disorders [31] [33]. We assert that RDoc is also incomplete without including certain body rhythms as one of its domains. In addition to helping the ill, the healthy could benefit from this understanding by harnessing the power body rhythms have to modulate the mind and unconscious bodily processes, potentially allowing one to control their mental state, endurance, and even such unconscious processes as immune reactions.

\section{The Default Mode Network}

In 1929, when electroencephalography was introduced, the brain was found to be continually active even when at rest [35]. Although this finding was initially rejected, evidence of such activity during undirected mental states began to accumulate [36]. Early research by Raichle and others leading to the technical identification and acceptance of a default mode of brain function [37] [38] [39] brought the default mode network into its own field of study [36]. A Modern view on the DMN suggests it largely provides a coherent phenomenal sense and cognitive representation of the self [40] [41] [42] [43] and that it varies in connectivity during differing states of self-referential functioning. It includes the brain areas of the posterior and anterior cingulate cortex, medial prefrontal cortex, inferior parietal cortex, hippocampal formation, and the precuneus [44] [45]. It was traditionally thought of as one system, but is now thought to be better conceptualized as composed of three integrated modules, each module contributing its own unique self-referential function [46]. The functional connectivity of these modules can change in opposing directions during certain states [47]. The three modules of the DMN include a frontal module (first-person perspective and agency), a posterior-right module (embodiment, autobiographical memory), and a posterior-left module (reflective-agency) [46]. The DMN is essential to normal mental functioning and abnormal DMN activity is evident in a variety of altered states [48] [49] and disorders including Alzheimer's [50], autism [51], schizophrenia [52], and Parkinson's [53].

Assuming that the default phenomenal state of being for a healthy person is the virtual replication of the internal and external world [21] [23] [54] [55], the sense of self and even the perception of having personal thoughts which are both strongly correlated with the DMN could can be considered a part of that model/simulation [56] [57] [58]. Thus, we assert the DMN plays a key role in modeling the virtual self within a greater virtual simulation. Metzinger has described the self as an illusion in that it is only a model of our mental and physical being with no technical basis in physical reality [56]. Trehub however acknowledges the simulation of the self, but insists it does have a real basis in a minimal self-referential neural system [57] which we assert would be the DMN. Functional connectivity of the resting state DMN is often defined by blood-oxygen level dependent (BOLD) signals [59]. The BOLD signal forms the basis of func- 
tional magnetic resonance imaging (fMRI) and is known to often correlate strongly with the bioelectric local field potentials [60]. The BOLD arises from electrical changes in oxy- to deoxyhemoglobin in the blood which reveals brain metabolism [59]. In healthy individuals, the patterns of BOLD activity in the DMN correlate with bioelectric oscillations such as those in the alpha band [61] [62]. In the resting state, the DMN exhibits BOLD fluctuations around $0.1 \mathrm{~Hz}$ which are correlated across distant regions of the brain [63].

Functional connectivity mapping of the DMN is thought to confuse identification of the DMN with respiratory and cardiac signals and that the respiratory related signals are artifacts that need to be separated [64]. We assert however that these signals are evidence of respiration/cardiac activity molding DMN connectivity. We aim to link the abnormal DMN activity seen in somatic maladies to altered somatic processes, thus revealing how the DMN is influenced and/or shaped by non-neural processes. Increased DMN integrity and activation in the anterior module is associated with depressive rumination and anxiety [65] [66]. Other somatic illnesses that result in altered DMN function include obesity [67], sleep apnea [68], respiratory disorders [69], gastrointestinal disorders [70], cardiac/vascular disorders [71], and others [72]. In our perspective, the abnormal DMN state of these disorders can be explained by the altered non-neural processes of the body which then influence the brain. We suggest these unconscious processes may include oscillations of oxygen and blood pressure in the vasculature, diverse sensory afferents, bio-molecular changes originating in the body, chemical signals and processes carried out by the micro-biome of the gut, and theoretical oscillations in bioelectricity which may reach the brain.

\section{The Central Autonomic Network}

The autonomic nervous system is unique in that its neurons exist in the body instead of the brain. Therefore, it has an important role in interfacing the two. We assert a much more intimate relationship between the autonomic nervous system and its cortical regulation networks and the DMN. The ANS is of top importance in everyday life and regulates a wide array of bodily functions [73]. We suggest that it also provides another mechanism for a holistic mind-body system allowing bodily activity to modulate if not underlie brain activity. Peripheral activity of the ANS is controlled by a variety of sites in the central nervous system, the central autonomic network (CAN) [73]. We stress the bi-directional nature and thus the existence of a unified system between this peripheral activity and the CAN and intrinsic brain networks. It is interesting that this network and the DMN share similar some anatomical sites including both angular gyri, both temporal poles, the posterior cingulate cortex, ventromedial prefrontal cortex, and precuneus [74]. In part because of this anatomical overlap, it has been suggested that the DMN is a high-level component of the CAN [75].

We and others suggest the overlap between the DMN and CAN may connect the self-referential activity to the bodily activity of the embodied but virtual 
self-model created by our brains. Activation levels in the CAN are generally stronger in the sympathetic state than in the parasympathetic [73]. We suggest that this is in part due to the strongly increased bodily activity during this state and that is it not just the brain sending increased activity to the body however, but the somatic activity potentially underlying neural activity is stimulating increased activity in the brain. The DMN is correlated with parasympathetic functions and anatomical localizations of the CAN and for the most part anti-correlated with sympathetic functioning and localization [73]. The parasympathetic division is focused on internal physiological function while the sympathetic state is purposed for external task-positive activity. Thus, it would make sense that the DMN, being a self-referential cognitive system, would be associated with self-based activity of the parasympathetic instead of the environmentally focused sympathetic state [36]. We will discuss how this may indicate a mechanism for somatic shaping of DMN function.

\section{Vascular Dynamics and Cognition}

Data generated from certain types of brain imaging such as functional magnetic resonance imaging convey hemodynamic changes that imply metabolic demand due to neural activity [76]. Although the local vascular changes that are observed in the brain during neural activity have been traditionally thought to be a unidirectional system modulated by neural activity, recent research is showing that vascular dynamics such as vasomotion (low-frequency oscillations in capillary radius) modulate and precede neuronal firing [29] [77] [78], and thus may be considered to be involved in cognition. Oscillations in oxygen and glucose influence neural ATP production, potentially entraining neural activity, likely assisted by astrocytes. Thus, a greater cognitive system may be formed when including the glia and vessels, providing a further means for the body to influence neural activity. Vessels in the brain receive blood from the body, and the number of these cerebral vessels is estimated to match the number of neurons in the brain at 100 billion [79], with most being finely dispersed capillaries [29]. Hemodynamic activity of these vessels occurs before the corresponding neural activity [76], in part suggesting that vessels play an important role in computation [77] and that the neuro-glial-vessel complex could be the most fundamental cognitive unit of the brain [78].

The system described not only consists of interactions between the neurons and capillary vessels, but the capillaries that feed the neurons interact among themselves via vaso-mediators [80] which are chemical signals underlying vascular changes [29]. Vascular dysfunctions related to these dynamics are associated with cognitive impairments [81]. Much more research is needed, but current research suggests that vascular dynamics do indeed play a key role in fundamental neural activity. This research has described an intimate connection between the slow hemodynamic BOLD oscillations and the fast bioelectric oscillations in the brain [82] [83] [84]. Resting state functional connectivities of the 
brain such as those of the DMN which are not understood through anatomical connections alone may instead be understood instead via vascular dynamics [59]. Collective, intrinsic, contractile oscillations of the vessel walls, vasomotion, occur around the $0.1 \mathrm{~Hz}$ of BOLD oscillations [85] which may tie them to the DMN.

If the vascular network has been left out of the current understanding of information processing in the brain, then it may be that there are further components left out of current models of fundamental cognitive systems which largely only consider neuronal systems. A greater model of a more global system may be formed from this neuro-glia-vessel cognitive system by including rhythms of the heart and lungs. Similar to how vascular rhythms are proposed to influence neural activity, by modulating oxygen, glucose, temperature, and lactate [29] [77], respiratory and cardiac rhythms would also influence the delivery of these resources to neurons. Oscillations of these bodily rhythms could thus modulate neural oscillations via oscillating metabolic resources delivered by the vessels. While individual neurons and their glial partners can influence local vascular activity [78], body rhythms would be best suited to modulate, entrain, or even underlie vascular dynamics on a global level. Similar to how low frequency bioelectric activity is shown to group, modulate, and entrain the more localized high frequency activity [16] [86] [87] [88], we posit that the even lower frequency cardiorespiratory and possibly other rhythms of the body could have similar entraining effects on the low frequency bioelectric oscillations.

\section{Rhythms of the Body to Rhythms of the Brain}

Not only does the state of the mind affect the body, but the physiological state of the body can influence various aspects of mental activity such as attention and memory encoding [89] [90]. Respiration in relation to brain activity has a long history of therapeutic application; however, the physiology of this link remains a mystery [16]. Respiration control is a fundamental aspect of ancient meditative practice [91], and is thought to be the main candidate underlying benefits from meditative practice [92]. These benefits include improvements in immunological functioning [93], general physical function and health [94], cardiopulmonary health [95], stress-resistance, attention control, and general cognitive functioning [96]. The dominant breathing technique across traditions is the slow-rate, deep inhalation style [92].

Although no direct explanation for the link between respiration and cognition has been founded, several authors have posited various ways of how the well-known effects respiration modulation can have on this mind arise [91] [92] [97] [98]. Ourselves and others suggest that respiratory sensory inputs (potentially all sensory inputs) and other respiratory mechanisms modulate and even in many senses underlie [20] the bioelectric neuronal synchronization from which consciousness is suggested to emerge [99]. Respiration is special when it comes to the body-mind system as unlike most bodily rhythms, we have the power to 
consciously control its activity. The Respiration rhythm is indeed observed to entrain bioelectric oscillations across the rodent and human brain (Figure 2) and is suggested to aid in long-range communication due to its global effects [100].

Gas exchange via the lungs is one of the most important physiological functions of the body, but there are many effects on mental and somatic state by respiration that cannot be explained by this gas exchange. One avenue for the effects of respiration is its influence on the heart and heart-rate variability. This relationship links respiration to blood flow and blood pressure changes in the brain which may modulate neural activity. Slow, deep breathing in particular has shown to immediately reduce blood pressure and heart rate [102]. Unexplained immediate effects due to respiration (inhalation vs. exhalation) include changes in motor control/force [103] [104], reaction time to sensory detection [17], and perception of pain [105]. The vagus nerve has been asserted to be the medium for respiratory-cognitive effects as this cranial nerve is modulated by respiration, being stimulated by slow breathing cycles [106]. The vagus nerve is mostly afferent, conveying to the CNS the homeostatic state of the viscera [107]. While this is likely an important medium for these effects, we assert a stronger role of other mediums we will discuss.

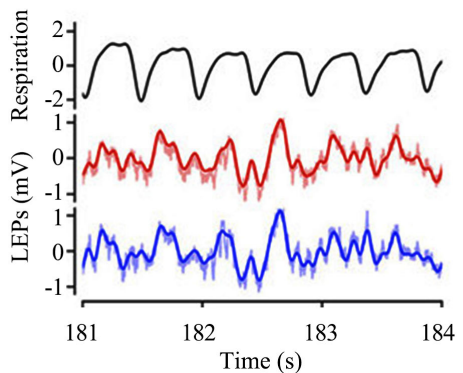

(a)

Piriform cortex

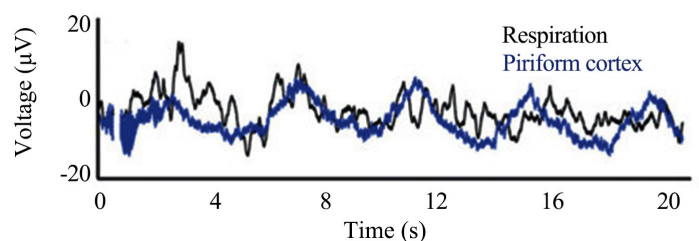

(c)

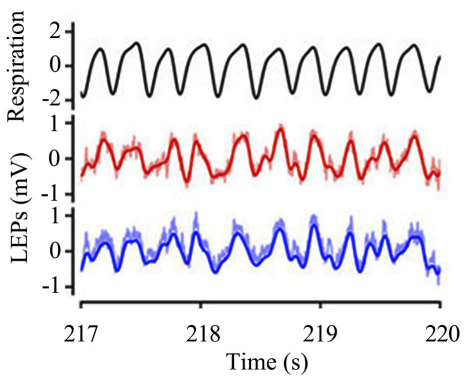

(b)

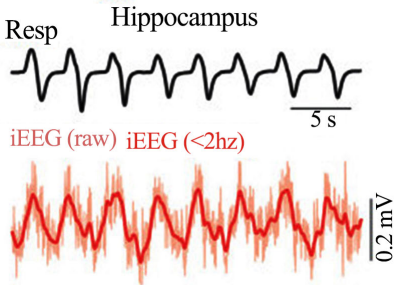

(d)

Figure 2. Respiration Entrained Brain Rhythms. These panels reveal simultaneous recordings of respiration and various bioelectric brain rhythms from across the rodent and human brains. These clearly illustrate that these respiratory patterns entrain brain activity. Panel a contains two graphs that show simultaneous recordings of respiration depth and corresponding brain activity in two areas of the somatosensory cortex of the awake mouse. One graph (right) shows a faster respiration rate which results in higher frequency bioelectric oscillations than the slower rate (left). Panel b shows human respiration rate and simultaneous recording from the Piriform cortex. Panel $\mathrm{c}$ shows Human respiration rate compared to simultaneous recording from the hippocampus. Rodent recordings adapted from [101]. Human recordings adapted from [100] (Copyright permissions obtained from Copyright Clearance Center ()). 
A close connection between the heart and the brain may reveal the nature of our association to many mental states with it. In many cultures and since ancient times, the heart has been metaphorically and literally attributed to certain emotions, such as love, and altruistic acts [108]. The heart is sometimes referred to as the "little brain" and has its own intrinsic network of neural plexuses and ganglia heavily influencing its functioning and afferent signaling the brain [109]. This intrinsic network even exhibits memory [109]. The heart actually sends more afferent signals to the brain than efferent signals it receives from it. Having a "broken heart" (Takotsubo Syndrome) can be caused by severe negative emotional and stressful states and can disrupt functional connectivity of the DMN [110]. This may suggest that the heart plays a critical role in DMN function.

Although the DMN has been strongly associated with the $0.1 \mathrm{~Hz}$ BOLD oscillations as discussed, it has more recently been associated with similar frequency infra-slow bioelectric oscillations recorded in the electroencephalogram (EEG) [111]. These infra-slow bioelectric oscillations have themselves been asserted to be extraneuronal in origin [112], and a proposed to reflect the same underlying neurophysiological phenomenon [111]. Fluctuations of arterial blood pressure (Mayer Waves) are also $0.1 \mathrm{~Hz}$ and correlated with the hemodynamic oscillations of the BOLD signal [113]. These hemodynamic oscillations are indeed strongly suggested to generate these infra slow bioelectric oscillations and this are an electrical counterpart to the hemodynamic activity [112]. It is also suggested that other spectra of bioelectric oscillations also have a hemodynamic counterpart [112]. This would in part explain the correlation of body rhythms with the bioelectric oscillations of the DMN and may further suggest in what manner body rhythms may influence the shaping of DMN connectivity. Figure 3 shows the bioelectric and hemodynamic measurements described.

Due to the suggested nature of how vascular dynamics underlie neural dynamics, variations in breathing style may exert the well-studied modulatory effects on mind via these vascular dynamics. We assert that via these vascular dynamics, metabolic activity of the neuro-glial-vessel complex could help shape intrinsic brain networks such as the DMN. The oscillatory activity of individual neurons is fundamental to the bioelectric frequencies of neural assemblies [114]. Thus, these metabolic oscillations may form a basis for widespread and dynamic bioelectric oscillations to be formed. As discussed, the DMN is highly correlated with parasympathetic activity. Considering a holistic system where the body and mind significantly influence each other, we suggest parasympathetic activity of the body initiated by the brain may in turn shape brain activity. In light of the research on bodily and neural rhythms, we assert that the slow respiratory rhythms stimulated by parasympathetic activity shape the BOLD oscillations which underlie DMN connectivity via vascular and other dynamics discussed. The slowed respiratory cycle during the parasympathetic state drive BOLD oscillations to a slower state and thus shape DMN connectivity. The fast respiration frequencies of the sympathetic system may functionally disconnect the DMN and promote task-positive networks via modulation of BOLD dynamics as well. 


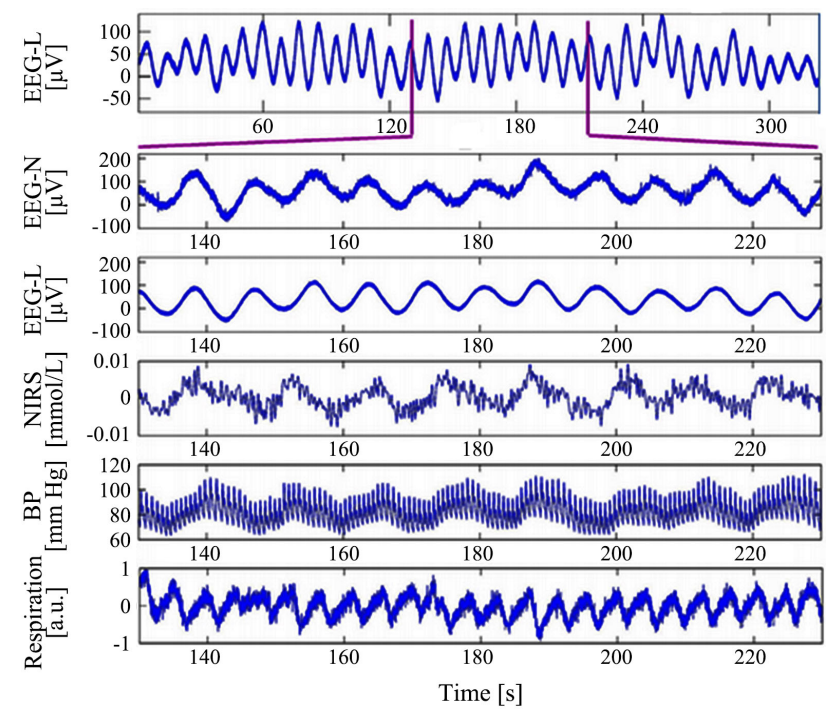

Figure 3. Bioelectric and Hemodynamic $0.1 \mathrm{~Hz}$ Oscillations in a Human Subject. These measurements correlate hemodynamic fluctuations with those of the EEG. The first Panel shows a greater time span which is shortened in the following panels. The two EEG panels are from the same electrode however EEG-N is nose referenced while EEG-L is Laplacian transformed. NIRS (Near-Infrared Spectroscopy) measures hemodynamic activity as seen in the BOLD response. BP is arterial blood pressure. Figure from [112] (Copyright permissions obtained from Copyright Clearance Center $\odot$ ).

\section{A Novel Body Rhythm}

Adding to the current literature describing how non-neural activity may underlie neural activity, we propose a novel theory on relationship between the body and mind. We propose that bioelectricity across all cells, not only the sensory afferents feeding to the CNS, are one major medium by which the body modulates the mind. We thus suggest the bioelectric, unified metastable continuum, an operational architecture of multiple levels of synchrony which is suggested to produce consciousness [115], actually extends to the body. While this continuum arises from dynamic and complex electric field interactions in the brain largely due to action potential generation, bioelectricity is a component of most if not all cells of the body [116]. Although somatic cells do not generate action potentials, many communicate electrically [116]. Bioelectric networks which perform cognitive acts in the brain may do the same in the body [117]. Non-neural changes in membrane potential are suggested to be a key component in this somatic computation [118]. While long-term somatic electrical activity has been found to be involved in development and regeneration [119], we assert short-term activity in the form of oscillations may have a deeper role as the base of the iceberg of mind.

We have previously proposed a novel body rhythm which may explain a variety of instantaneous effects observed due to respiration. This rhythm is the distribution of free electrons throughout body and brain from and generated by the lungs [120]. We have proposed that electrons are "harvested" from oxygen via redox reactions in the lungs during inspiration and travel via conduction to 
cells throughout the body where they attach to intracellular proteins and acids. In this way, inspiration modulates membrane potential in a rhythmic fashion identical to the rhythm of inspiration via hyperpolarization of the cellular membrane potential. Respiration does indeed appear to entrain neuronal membrane potential in this fashion (Figure 4). Upon, expiration, depolarization would occur as the electrical currents are drawn back out of every cell and released in carbon dioxide. This process would repeat upon each breath, creating a global rhythm across the body and brain which would homeostatically unify a variety of processes throughout the body-mind system.

If these currents exist, then they may work to shape intrinsic bioelectric activity of the brain such as the activity of the DMN. The dynamics and correlations between respiration and brain activity may better be described by this electrical current rhythm of the lungs than by the other rhythms described in this article which still play some role. These currents may form the base of a frequency based hierarchy of a bioelectric architecture proposed to be isomorphic to the conscious mind [28] [115]. As mentioned previously, slower frequency bioelectric activity is known to entrain faster activity. A bioelectric rhythm identical to the respiratory rhythm would thus provide a foundation for all faster frequency to build and would provide an explanation for the powerful effects respiration has on neural oscillations and bioelectric activity of the body such as heart rate variability. These electrical currents may also provide a very efficient energy delivery mechanism by providing electrons to cells throughout the body to be used for metabolic purposes. In testing this hypothesis, simultaneous monitoring of respiration pattern and cellular membrane potentials from across the body and brain would reveal if membrane potential oscillations do indeed match oscillations in respiration.

\section{Conclusions}

The current understanding of the DMN BOLD oscillations is that these infra slow oscillations around $0.1 \mathrm{~Hz}$ and the infra-slow bioelectric oscillations associated with them are associated with self-referential cognitive processing. Based on our review of literature including findings from cardiorespiratory physiology, the DMN oscillations provide a foundation for faster oscillations that are

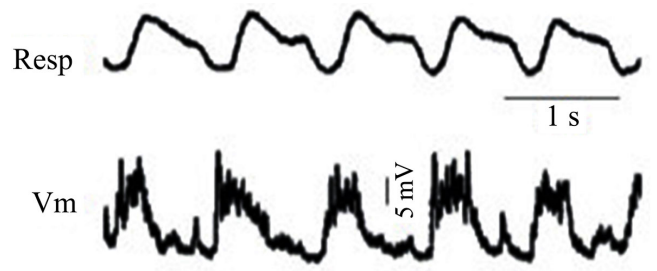

Figure 4. Entrainment of neuronal membrane potential by respiration. This image shows simultaneous recording of respiration depth and intracellular potential $(\mathrm{Vm})$ of a neuron in the piriform cortex of the anesthetized rat. This reveals that respiration entrainment of neural activity extends to the cellular level. Adapted from [100] (Copyright permissions obtained from Copyright Clearance Center ( )). 
involved with cognition. We have asserted a hypothesis on the basis of DMN and intrinsic brain activity as being founded on bodily rhythms. These DMN oscillations may create a virtual structure which is the basis of mind, a "self" within a 3D space which constitutes a replication of the internal and external world. This provides a virtual structure for the virtual isomorphism of the faster oscillations to build upon. The oscillations can also be considered "energy" oscillations as they further provide a metabolic and bioelectric foundation for higher oscillations and these DMN oscillations may primarily be conducted by astrocyte syncytium. We have asserted a novel coordination mechanism of neurovascular activity generated from the cardiorespiratory system that repolarizes and depolarizes such a neuro-glial complex. It is evident that the respiratory and other bodily rhythms have significant influence on intrinsic brain activity but this significance is debated. The exact role of unconscious visceral rhythms of the body and the influence of the peripheral nervous system on the formation and structure of central nervous system activity is not fully understood. Our hypothesis on the importance and potential mechanisms of such peripheral activity on the formation of mind may shed light on this mystery.

The brain needs significant energy for its functions and resting state networks; for example the DMN consumes a majority of this energy. For neural activity to be maintained, fast re-polarization mechanisms are required. We have asserted a mechanism that may provide this functionality based on respiration rhythm. The $0.1 \mathrm{~Hz}$ rhythm frequently is indistinguishable from respiratory rhythm. This rhythm is also noted to be associated with heart rate variability and other peripheral rhythms suggesting a mind body connection that is fundamental to intrinsic cognition and we have proposed that it provides energy and order for the faster rhythms to form in the form of rhythmic electrical currents originating in the lungs. It is important to recognize and further investigate the nature of this rhythm for a better understanding of mind and the functional origin and purpose of neural oscillations and this may reveal new understandings of the neuropathology of a variety of disorders.

\section{Conflicts of Interest}

The authors declare no conflicts of interest regarding the publication of this paper.

\section{References}

[1] Wilson, A.D. and Golonka, S. (2013) Embodied Cognition Is Not What You Think It Is. Frontiers in Psychology, 4, 58. https://doi.org/10.3389/fpsyg.2013.00058

[2] Wilson, R.A. and Foglia, L. (2017) Embodied Cognition. In: The Stanford Encyclopedia of Philosophy, Metaphysics Research Lab, Stanford University, Stanford. https://plato.stanford.edu/entries/embodied-cognition/

[3] Favela, L.H. (2014) Radical Embodied Cognitive Neuroscience: Addressing "Grand Challenges" of the Mind Sciences. Frontiers in Human Neuroscience, 8, 796. https://doi.org/10.3389/fnhum.2014.00796 
[4] Baluška, F. and Levin, M. (2016) On Having No Head: Cognition throughout Biological Systems. Frontiers in Psychology, 7, 902. https://doi.org/10.3389/fpsyg.2016.00902

[5] Turner, C.H., et al. (2002) Do Bone Cells Behave like a Neuronal Network? Calcified Tissue International, 70, 435-442. https://doi.org/10.1007/s00223-001-1024-Z

[6] Gundersen, K. (2016) Muscle Memory and a New Cellular Model for Muscle Atrophy and Hypertrophy. The Journal of Experimental Biology, 219, 235-242. https://doi.org/10.1242/jeb.124495

[7] Rosen, M.R. and Cohen, I.S. (2006) Cardiac Memory ... New Insights into Molecular Mechanisms. The Journal of Physiology, 570, 209-218. https://doi.org/10.1113/jphysiol.2005.097873

[8] Lobo, D., et al. (2013) A Linear-Encoding Model Explains the Variability of the Target Morphology in Regeneration. Journal of the Royal Society, Interface, 11, Article ID: 20130918. https://doi.org/10.1098/rsif.2013.0918

[9] Alvarez, L., et al. (2014) The Computational Sperm Cell. Trends in Cell Biology, 24, 198-207. https://doi.org/10.1016/j.tcb.2013.10.004

[10] Law, R. and Levin, M. (2015) Bioelectric Memory: Modeling Resting Potential Bistability in Amphibian Embryos and Mammalian Cells. Theoretical Biology \& Medical Modelling, 12, 22. https://doi.org/10.1186/s12976-015-0019-9

[11] Ekman, P., Levenson, R. and Friesen, W. (1983) Autonomic Nervous System Activity Distinguishes among Emotions. Science, 221, 1208-1210. https://doi.org/10.1126/science.6612338

[12] Liebeskind, B.J., Hillis, D.M. and Zakon, H.H. (2011) Evolution of Sodium Channels Predates the Origin of Nervous Systems in Animals. Proceedings of the National Academy of Sciences, 108, 9154-9159. https://doi.org/10.1073/pnas.1106363108

[13] Keijzer, F., van Duijn, M. and Lyon, P. (2013) What Nervous Systems Do: Early Evolution, Input-Output, and the Skin Brain Thesis. Adaptive Behavior, 21, 67-85. https://doi.org/10.1177/1059712312465330

[14] Heck, D.H., et al. (2017) Breathing as a Fundamental Rhythm of Brain Function. Frontiers in Neural Circuits, 10, 115. https://doi.org/10.3389/fncir.2016.00115

[15] Bordoni, B., et al. (2018) The Influence of Breathing on the Central Nervous System. Cureus, 10, e2724-e2724. https://doi.org/10.7759/cureus.2724

[16] Herrero, J.L., et al. (2018) Breathing above the Brain Stem: Volitional Control and Attentional Modulation in Humans. Journal of Neurophysiology, 119, 145-159. https://doi.org/10.1152/jn.00551.2017

[17] Zelano, C., et al. (2016) Nasal Respiration Entrains Human Limbic Oscillations and Modulates Cognitive Function. The Journal of Neuroscience, 36, 12448-12467. https://doi.org/10.1523/JNEUROSCI.2586-16.2016

[18] Lockmann, A.L.V., et al. (2016) A Respiration-Coupled Rhythm in the Rat Hippocampus Independent of Theta and Slow Oscillations. The Journal of Neuroscience, 36, 5338-5352. https://doi.org/10.1523/JNEUROSCI.3452-15.2016

[19] Breit, S., et al. (2018) Vagus Nerve as Modulator of the Brain-Gut Axis in Psychiatric and Inflammatory Disorders. Frontiers in Psychiatry, 9, 44. https://doi.org/10.3389/fpsyt.2018.00044

[20] Varga, S. and Heck, D.H. (2017) Rhythms of the Body, Rhythms of the Brain: Respiration, Neural Oscillations, and Embodied Cognition. Consciousness and Cognition, 56, 77-90. https://doi.org/10.1016/j.concog.2017.09.008 
[21] Fingelkurts, A.A., Fingelkurts, A.A. and Neves, C.F.H. (2010) Natural World Physical, Brain Operational, and Mind Phenomenal Space-Time. Physics of Life Reviews, 7, 195-249. https://doi.org/10.1016/j.plrev.2010.04.001

[22] Fingelkurts, A.A. and Fingelkurts, A.A. (2001) Operational Architectonics of the Human Brain Biopotential Field: Towards Solving the Mind-Brain Problem. Brain and Mind, 2, 261-296. https://doi.org/10.1023/A:1014427822738

[23] Jerath, R. and Beveridge, C. (2019) Multimodal Integration and Phenomenal Spatiotemporal Binding: A Perspective From the Default Space Theory. Frontiers in Integrative Neuroscience, 13, 2. https://doi.org/10.3389/fnint.2019.00002

[24] Specker Sullivan, L. (2018) Pure Experience and Disorders of Consciousness. AJOB Neuroscience, 9, 107-114. https://doi.org/10.1080/21507740.2018.1459931

[25] Edwards, S. (2006) Experiencing the Meaning of Breathing. Indo-Pacific Journal of Phenomenology, 6, 1-13. https://doi.org/10.1080/20797222.2006.11433911

[26] Kox, M., et al. (2014) Voluntary Activation of the Sympathetic Nervous System and Attenuation of the Innate Immune Response in Humans. Proceedings of the National Academy of Sciences, 111, 7379-7384.

https://doi.org/10.1073/pnas.1322174111

[27] Grossman, L.C.D. and Christensen, L. (2017) On Combat: The Psychology and Physiology of Deadly Conflict in War and in Peace.

[28] Jerath, R. and Crawford, M.W. (2015) Layers of Human Brain Activity: A Functional Model Based on the Default Mode Network and Slow Oscillations. Frontiers in Human Neuroscience, 9, 248. https://doi.org/10.3389/fnhum.2015.00248

[29] Philips, R.T., Chhabria, K. and Chakravarthy, V.S. (2016) Vascular Dynamics Aid a Coupled Neurovascular Network Learn Sparse Independent Features: A Computational Model. Frontiers in Neural Circuits, 10, 7.

https://doi.org/10.3389/fncir.2016.00007

[30] Fingelkurts, A.A. and Fingelkurts, A.A. (2019) Brain Space and Time in Mental Disorders: Paradigm Shift in Biological Psychiatry. The International Journal of Psychiatry in Medicine, 54, 53-63. https://doi.org/10.1177/0091217418791438

[31] Kirmayer, L.J. and Crafa, D. (2014) What Kind of Science for Psychiatry? Frontiers in Human Neuroscience, 8, 435-435. https://doi.org/10.3389/fnhum.2014.00435

[32] Buckholtz, J.W. and Meyer-Lindenberg, A. (2012) Psychopathology and the Human Connectome: Toward a Transdiagnostic Model of Risk for Mental Illness. Neuron, 74, 990-1004. https://doi.org/10.1016/j.neuron.2012.06.002

[33] Casey, B.J., et al. (2013) DSM-5 and RDoC: Progress in Psychiatry Research? Nature Reviews. Neuroscience, 14, 810-814. https://doi.org/10.1038/nrn3621

[34] Walter, H. (2013) The Third Wave of Biological Psychiatry. Frontiers in Psychology, 4, 582-582. https://doi.org/10.3389/fpsyg.2013.00582

[35] Berger, H. (1929) Über das elektrenkephalogramm des menschen. European Archives of Psychiatry and Clinical Neuroscience, 87, 527-570. https://doi.org/10.1007/BF01797193

[36] Buckner, R.L., Andrews-Hanna, J.R. and Schacter, D.L. (2008) The Brain's Default Network. Annals of the New York Academy of Sciences, 1124, 1-38. https://doi.org/10.1196/annals.1440.011

[37] Gusnard, D.A., et al. (2001) Medial Prefrontal Cortex and Self-Referential Mental Activity: Relation to a Default Mode of Brain Function. Proceedings of the National Academy of Sciences of the United States of America, 98, 4259-4264. https://doi.org/10.1073/pnas.071043098 
[38] Gusnard, D.A. and Raichle, M.E. (2001) Searching for a Baseline: Functional Imaging and the Resting Human Brain. Nature Reviews Neuroscience, 2, 685-694. https://doi.org/10.1038/35094500

[39] Raichle, M.E., et al. (2001) A Default Mode of Brain Function. Proceedings of the National Academy of Sciences, 98, 676-682. https://doi.org/10.1073/pnas.98.2.676

[40] Fingelkurts, A.A., et al. (2012) DMN Operational Synchrony Relates to Self-Consciousness: Evidence from Patients in Vegetative and Minimally Conscious States. The Open Neuroimaging Journal, 6, 55-68. https://doi.org/10.2174/1874440001206010055

[41] Fingelkurts, A.A., et al. (2016) The Chief Role of Frontal Operational Module of the Brain Default Mode Network in the Potential Recovery of Consciousness from the Vegetative State: A Preliminary Comparison of Three Case Reports. The Open Neuroimaging Journal, 10, 41-51. https://doi.org/10.2174/1874440001610010041

[42] Schilbach, L., et al. (2008) Minds at Rest? Social Cognition as the Default Mode of Cognizing and Its Putative Relationship to the "Default System" of the Brain. Consciousness and Cognition, 17, 457-467. https://doi.org/10.1016/j.concog.2008.03.013

[43] Buckner, R.L. and Carroll, D.C. (2007) Self-Projection and the Brain. Trends in Cognitive Sciences, 11, 49-57. https://doi.org/10.1016/j.tics.2006.11.004

[44] Brueggen, K., et al. (2017) Early Changes in Alpha Band Power and DMN BOLD Activity in Alzheimer's Disease: A Simultaneous Resting State EEG-fMRI Study. Frontiers in Aging Neuroscience, 9, 319. https://doi.org/10.3389/fnagi.2017.00319

[45] Raichle, M.E., et al. (2001) A Default Mode of Brain Function. Proceedings of the National Academy of Sciences of the United States of America, 98, 676-682. https://doi.org/10.1073/pnas.98.2.676

[46] Fingelkurts, A.A., Fingelkurts, A.A. and Kallio-Tamminen, T. (2016) Trait Lasting Alteration of the Brain Default Mode Network in Experienced Meditators and the Experiential Selfhood. Self and Identity, 15, 381-393. https://doi.org/10.1080/15298868.2015.1136351

[47] Fingelkurts, A.A., Fingelkurts, A.A. and Kallio-Tamminen (2016) Long-Term Meditation Training Induced Changes in the Operational Synchrony of Default Mode Network Modules during a Resting State. Cognitive Processing, 17, 27. https://doi.org/10.1007/s10339-015-0743-4

[48] Vanhaudenhuyse, A., et al. (2010) Default Network Connectivity Reflects the Level of Consciousness in Non-Communicative Brain-Damaged Patients. Brain, 133, 161-171. https://doi.org/10.1093/brain/awp313

[49] Palhano-Fontes, F., et al. (2015) The Psychedelic State Induced by Ayahuasca Modulates the Activity and Connectivity of the Default Mode Network. PLoS ONE, 10, e0118143. https://doi.org/10.1371/journal.pone.0118143

[50] Wu, X., et al. (2011) Altered Default Mode Network Connectivity in Alzheimer's Disease-A Resting Functional MRI and Bayesian Network Study. Human Brain Mapping, 32, 1868-1881. https://doi.org/10.1002/hbm.21153

[51] Padmanabhan, A., et al. (2017) The Default Mode Network in Autism. Biological Psychiatry. Cognitive Neuroscience and Neuroimaging, 2, 476-486. https://doi.org/10.1016/j.bpsc.2017.04.004

[52] Wang, H., et al. (2015) Evidence of a Dissociation Pattern in Default Mode Subnetwork Functional Connectivity in Schizophrenia. Scientific Reports, 5, Article No. 14655. https://doi.org/10.1038/srep14655

[53] van Eimeren, T., et al. (2009) Dysfunction of the Default Mode Network in Parkin- 
son Disease: A Functional Magnetic Resonance Imaging Study. Archives of Neurology, 66, 877-883. https://doi.org/10.1001/archneurol.2009.97

[54] Revonsuo, A. (2006) Inner Presence: Consciousness as a Biological Phenomenon. MIT Press, Cambridge.

[55] Grush, R. (2004) The Emulation Theory of Representation: Motor Control, Imagery, and Perception. Behavioral and Brain Sciences, 27, 377-442. https://doi.org/10.1017/S0140525X04000093

[56] Metzinger, T. (2003) Being No-One. MIT Press, Cambridge. https://doi.org/10.7551/mitpress/1551.001.0001

[57] Trehub, A. (2007) Space, Self, and the Theater of Consciousness. Consciousness and Cognition, 16, 310-330. https://doi.org/10.1016/j.concog.2006.06.004

[58] Hesslow, G. (2002) Conscious Thought as Simulation of Behaviour and Perception. Trends in Cognitive Sciences, 6, 242-247. https://doi.org/10.1016/S1364-6613(02)01913-7

[59] Mateo, C., et al. (2017) Entrainment of Arteriole Vasomotor Fluctuations by Neural Activity Is a Basis of Blood-Oxygenation-Level-Dependent "Resting-State" Connectivity. Neuron, 96, 936-948.e3. https://doi.org/10.1016/j.neuron.2017.10.012

[60] Ekstrom, A. (2010) How and When the fMRI BOLD Signal Relates to Underlying Neural Activity: The Danger in Dissociation. Brain Research Reviews, 62, 233-244. https://doi.org/10.1016/j.brainresrev.2009.12.004

[61] Scheeringa, R., et al. (2012) EEG $\alpha$ Power Modulation of fMRI Resting-State Connectivity. Brain Connectivity, 2, 254-264. https://doi.org/10.1089/brain.2012.0088

[62] Jann, K., et al. (2010) Topographic Electrophysiological Signatures of fMRI Resting State Networks. PLoS ONE, 5, e12945. https://doi.org/10.1371/journal.pone.0012945

[63] Fox, M.D. and Raichle, M.E. (2007) Spontaneous Fluctuations in Brain Activity Observed with Functional Magnetic Resonance Imaging. Nature Reviews Neuroscience, 8, 700. https://doi.org/10.1038/nrn2201

[64] Birn, R.M., Murphy, K. and Bandettini, P.A. (2008) The Effect of Respiration Variations on Independent Component Analysis Results of Resting State Functional Connectivity. Human Brain Mapping, 29, 740-750.

https://doi.org/10.1002/hbm.20577

[65] Hamilton, J.P., et al. (2015) Depressive Rumination, the Default-Mode Network, and the Dark Matter of Clinical Neuroscience. Biological Psychiatry, 78, 224-230. https://doi.org/10.1016/j.biopsych.2015.02.020

[66] Coutinho, J.F., et al. (2016) Default Mode Network Dissociation in Depressive and Anxiety States. Brain Imaging and Behavior, 10, 147-157. https://doi.org/10.1007/s11682-015-9375-7

[67] Beyer, F., et al. (2017) Higher Body Mass Index Is Associated with Reduced Posterior Default Mode Connectivity in Older Adults. Human Brain Mapping, 38, 3502-3515. https://doi.org/10.1002/hbm.23605

[68] Li, H., et al. (2015) Abnormal Resting-State Functional Connectivity within the Default Mode Network Subregions in Male Patients with Obstructive Sleep Apnea. Neuropsychiatric Disease and Treatment, 12, 203-212. https://doi.org/10.2147/NDT.S97449

[69] Li, Q.-G., et al. (2018) Alterations of Resting-State Functional Network Centrality in Patients with Asthma: Evidence from a Voxel-Wise Degree Centrality Analysis. NeuroReport, 29, 1151-1156. https://doi.org/10.1097/WNR.0000000000001087 
[70] Kano, M., et al. (2018) Understanding Neurogastroenterology from Neuroimaging Perspective: A Comprehensive Review of Functional and Structural Brain Imaging in Functional Gastrointestinal Disorders. Journal of Neurogastroenterology and Motility, 24, 512-527. https://doi.org/10.5056/jnm18072

[71] Silva, D.S., et al. (2018) Default Mode Network Disruption in Stroke-Free Patients with Atrial Fibrillation. Cerebrovascular Diseases, 45, 78-84. https://doi.org/10.1159/000486689

[72] Cocozza, S., et al. (2018) Default Mode Network Modifications in Fabry Disease: A Resting-State fMRI Study with Structural Correlations. Human Brain Mapping, 39, 1755-1764. https://doi.org/10.1002/hbm.23949

[73] Beissner, F., et al. (2013) The Autonomic Brain: An Activation Likelihood Estimation Meta-Analysis for Central Processing of Autonomic Function. The Journal of Neuroscience. The Official Journal of the Society for Neuroscience, 33, 10503-10511. https://doi.org/10.1523/JNEUROSCI.1103-13.2013

[74] Bär, K.-J., et al. (2015) Relation of Autonomic Measures to the Default Mode Network. Autonomic Neuroscience: Basic and Clinical, 192, 11. https://doi.org/10.1016/j.autneu.2015.07.282

[75] Smith, R., et al. (2017) The Hierarchical Basis of Neurovisceral Integration. Neuroscience \& Biobehavioral Reviews, 75, 274-296. https://doi.org/10.1016/j.neubiorev.2017.02.003

[76] Sirotin, Y.B. and Das, A. (2009) Anticipatory Haemodynamic Signals in Sensory Cortex Not Predicted by Local Neuronal Activity. Nature, 457, 475-479. https://doi.org/10.1038/nature07664

[77] Moore, C.I. and Cao, R. (2008) The Hemo-Neural Hypothesis: On the Role of Blood Flow in Information Processing. Journal of Neurophysiology, 99, 2035-2047. https://doi.org/10.1152/jn.01366.2006

[78] Chander, B.S. and Chakravarthy, V.S. (2012) A Computational Model of Neuro-Glio-Vascular Loop Interactions. PLoS ONE, 7, e48802. https://doi.org/10.1371/journal.pone.0048802

[79] Quaegebeur, A., Lange, C. and Carmeliet, P. (2011) The Neurovascular Link in Health and Disease: Molecular Mechanisms and Therapeutic Implications. Neuron, 71, 406-424. https://doi.org/10.1016/j.neuron.2011.07.013

[80] Vanhoutte, P.M. and Mombouli, J.-V. (1996) Vascular Endothelium: Vasoactive Mediators. Progress in Cardiovascular Diseases, 39, 229-238.

https://doi.org/10.1016/S0033-0620(96)80003-X

[81] Di Marco, L., et al. (2015) Is Vasomotion in Cerebral Arteries Impaired in Alzheimer's Disease? Journal of Alzheimer's Disease, 46, 35-53. https://doi.org/10.3233/JAD-142976

[82] Yuan, H., et al. (2012) Spatiotemporal Dynamics of the Brain at Rest-Exploring EEG Microstates as Electrophysiological Signatures of BOLD Resting State Networks. Neuroimage, 60, 2062-2072. https://doi.org/10.1016/j.neuroimage.2012.02.031

[83] Pan, W.-J., et al. (2013) Infraslow LFP Correlates to Resting-State fMRI BOLD Signals. NeuroImage, 74, 288-297. https://doi.org/10.1016/j.neuroimage.2013.02.035

[84] Golkowski, D., et al. (2017) Coherence of BOLD Signal and Electrical Activity in the Human Brain during Deep Sevoflurane Anesthesia. Brain and Behavior, 7, e00679. https://doi.org/10.1002/brb3.679

[85] Obrig, H., et al. (2000) Spontaneous Low Frequency Oscillations of Cerebral He- 
modynamics and Metabolism in Human Adults. NeuroImage, 12, 623-639. https://doi.org/10.1006/nimg.2000.0657

[86] Sirota, A. and Buzsaki, G. (2005) Interaction between Neocortical and Hippocampal Networks via Slow Oscillations. Thalamus \& Related Systems, 3, 245-259. https://doi.org/10.1017/S1472928807000258

[87] Buzsaki, G. and Wang, X.J. (2012) Mechanisms of Gamma Oscillations. Annual Review of Neuroscience, 35, 203-225. https://doi.org/10.1146/annurev-neuro-062111-150444

[88] Steriade, M. (2006) Grouping of Brain Rhythms in Corticothalamic Systems. Neuroscience, 137, 1087-1106. https://doi.org/10.1016/j.neuroscience.2005.10.029

[89] Sie, J.-H., et al. (2019) Altered Central Autonomic Network in Baseball Players: A Resting-State fMRI Study. Scientific Reports, 9, Article No. 110. https://doi.org/10.1038/s41598-018-36329-9

[90] Critchley, H.D., Eccles, J. and Garfinkel, S.N. (2013) Chapter 6 Interaction between Cognition, Emotion, and the Autonomic Nervous System. In: Buijs, R.M. and Swaab, D.F., Eds., Handbook of Clinical Neurology, Elsevier, Amsterdam, 59-77. https://doi.org/10.1016/B978-0-444-53491-0.00006-7

[91] Melnychuk, M.C., et al. (2018) Coupling of Respiration and Attention via the Locus Coeruleus: Effects of Meditation and Pranayama. Psychophysiology, 55, e13091. https://doi.org/10.1111/psyp.13091

[92] Gerritsen, R.J.S. and Band, G.P.H. (2018) Breath of Life: The Respiratory Vagal Stimulation Model of Contemplative Activity. Frontiers in Human Neuroscience, 12, Article No. 397. https://doi.org/10.3389/fnhum.2018.00397

[93] Black, D.S., et al. (2013) Yogic Meditation Reverses NF- $\kappa$ B and IRF-Related Transcriptome Dynamics in Leukocytes of Family Dementia Caregivers in a Randomized Controlled Trial. Psychoneuroendocrinology, 38, 348-355. https://doi.org/10.1016/j.psyneuen.2012.06.011

[94] Büssing, A., et al. (2012) Effects of Yoga on Mental and Physical Health: A Short Summary of Reviews. Evidence-Based Complementary and Alternative Medicine, 2012, Article ID: 165410. https://doi.org/10.1155/2012/165410

[95] Ospina, M.B., et al. (2007) Meditation Practices for Health: State of the Research. Evidence Report/ Technology Assessment, 155, 1-263.

[96] Eberth, J. and Sedlmeier, P. (2012) The Effects of Mindfulness Meditation: A Meta-Analysis. Mindfulness, 3, 174-189. https://doi.org/10.1007/s12671-012-0101-x

[97] Jerath, R., Beveridge, C. and Barnes, V.A. (2019) Self-Regulation of Breathing as an Adjunctive Treatment of Insomnia. Frontiers in Psychiatry, 9, 780. https://doi.org/10.3389/fpsyt.2018.00780

[98] Jerath, R., et al. (2015) Self-Regulation of Breathing as a Primary Treatment for Anxiety. Applied Psychophysiology and Biofeedback, 40, 107-115. https://doi.org/10.1007/s10484-015-9279-8

[99] Fingelkurts, A.A., Fingelkurts, A.A. and Neves, C.F.H. (2013) Consciousness as a Phenomenon in the Operational Architectonics of Brain Organization: Criticality and Self-Organization Considerations. Chaos, Solitons \& Fractals, 55, 13-31. https://doi.org/10.1016/j.chaos.2013.02.007

[100] Tort, A.B.L., Brankačk, J. and Draguhn, A. (2018) Respiration-Entrained Brain Rhythms Are Global But Often Overlooked. Trends in Neurosciences, 41, 186-197. https://doi.org/10.1016/j.tins.2018.01.007

[101] Ito, J., et al. (2014) Whisker Barrel Cortex Delta Oscillations and Gamma Power in 
the Awake Mouse Are Linked to Respiration. Nature Communications, 5, 3572. https://doi.org/10.1038/ncomms4572

[102] Pramanik, T., et al. (2009) Immediate Effect of Slow Pace Bhastrika Pranayama on Blood Pressure and Heart Rate. The Journal of Alternative and Complementary Medicine, 15, 293-295. https://doi.org/10.1089/acm.2008.0440

[103] Li, S. and Laskin, J.J. (2006) Influences of Ventilation on Maximal Isometric Force of the Finger Flexors. Muscle \& Nerve, 34, 651-655. https://doi.org/10.1002/mus.20592

[104] Li, S. and Rymer, W.Z. (2011) Voluntary Breathing Influences Corticospinal Excitability of Nonrespiratory Finger Muscles. Journal of Neurophysiology, 105, 512-521. https://doi.org/10.1152/jn.00946.2010

[105] Iwabe, T., Ozaki, I. and Hashizume, A. (2014) The Respiratory Cycle Modulates Brain Potentials, Sympathetic Activity, and Subjective Pain Sensation Induced by Noxious Stimulation. Neuroscience Research, 84, 47-59. https://doi.org/10.1016/j.neures.2014.03.003

[106] Chang, R.B., et al. (2015) Vagal Sensory Neuron Subtypes that Differentially Control Breathing. Cell, 161, 622-633. https://doi.org/10.1016/j.cell.2015.03.022

[107] Agostoni, E., et al. (1957) Functional and Histological Studies of the Vagus Nerve and Its Branches to the Heart, Lungs and Abdominal Viscera in the Cat. The Journal of Physiology, 135, 182-205. https://doi.org/10.1113/jphysiol.1957.sp005703

[108] Powell, P.A., et al. (2018) Heart versus Head: Differential Bodily Feedback Causally Alters Economic Decision-Making. Quarterly Journal of Experimental Psychology, 71, 1949-1959. https://doi.org/10.1080/17470218.2017.1373359

[109] Ardell, J. (2004) Intrathoracic Neuronal Regulation of Cardiac Function. In: Ardell, J.L. and Armour, J.A. Eds., Basic and Clinical Neurocardiology, Oxford University Press, New York, 118-152.

[110] Templin, C., et al. (2019) Altered Limbic and Autonomic Processing Supports Brain-Heart Axis in Takotsubo Syndrome. European Heart Journal, 40, 1183-1187. https://doi.org/10.1093/eurheartj/ehz068

[111] Hiltunen, T., et al. (2014) Infra-Slow EEG Fluctuations Are Correlated with Resting-State Network Dynamics in fMRI. The Journal of Neuroscience, 34, 356-362. https://doi.org/10.1523/JNEUROSCI.0276-13.2014

[112] Nikulin, V.V., et al. (2014) Monochromatic Ultra-Slow $(\sim 0.1 \mathrm{~Hz})$ Oscillations in the Human Electroencephalogram and Their Relation to Hemodynamics. NeuroImage, 97, 71-80. https://doi.org/10.1016/j.neuroimage.2014.04.008

[113] Pfurtscheller, G., et al. (2011) About the Stability of Phase Shifts between Slow Oscillations Around $0.1 \mathrm{~Hz}$ in Cardiovascular and Cerebral Systems. IEEE Transactions on Biomedical Engineering, 58, 2064-2071. https://doi.org/10.1109/TBME.2011.2134851

[114] Başar, E. and Düzgün, A. (2016) The CLAIR Model: Extension of Brodmann Areas Based on Brain Oscillations and Connectivity. International Journal of Psychophysiology, 103, 185-198. https://doi.org/10.1016/j.ijpsycho.2015.02.018

[115] Fingelkurts, A.A., Fingelkurts, A.A. and Neves, C.F.H. (2009) Phenomenological Architecture of a Mind and Operational Architectonics of the Brain: The Unified Metastable Continuum. New Mathematics and Natural Computation, 5, 221-244. https://doi.org/10.1142/S1793005709001258

[116] McCaig, C.D., et al. (2005) Controlling Cell Behavior Electrically: Current Views and Future Potential. Physiological Reviews, 85, 943-978. 
https://doi.org/10.1152/physrev.00020.2004

[117] Levin, M. and Stevenson, C.G. (2012) Regulation of Cell Behavior and Tissue Patterning by Bioelectrical Signals: Challenges and Opportunities for Biomedical Engineering. Annual Review of Biomedical Engineering, 14, 295-323. https://doi.org/10.1146/annurev-bioeng-071811-150114

[118] Funk, R. (2013) Ion Gradients in Tissue and Organ Biology. Biological Systems Open Access, 2, 105.

[119] Levin, M. (2014) Molecular Bioelectricity: How Endogenous Voltage Potentials Control Cell Behavior and Instruct Pattern Regulation in Vivo. Molecular Biology of the Cell, 25, 3835-3850. https://doi.org/10.1091/mbc.e13-12-0708

[120] Jerath, R. and Beveridge, C. (2018) Novel Bioelectric Mechanisms and Functional Significance of Peripheral and Central Entrainment by Respiration. World Journal of Neuroscience, 8, 480-500. https://doi.org/10.4236/wjns.2018.84038 